\section{The Chemical Components of Tobacco and Tobacco Smoke}

by Alan Rodgman and Thomas $\mathrm{A}$. Perfetti: CRC Press, Boca Raton, FI, USA, 2009, pp 928,

ISBN 978-01-4200-7883-1.

Price: GBP 191.00, USD 299.95

The arrival of this book generated a response full of misgivings. With more than 1,750 pages I could see a repetitive strain injury occurring merely by turning the pages. It was with some considerable relief therefore that a quick inspection revealed that this was a reference book and was packed full of tables. It is essentially a summary of the chemical status quo for the tobacco industry. I was thus able to formulate a strategy for assessment whereby I selected a set of chapters and evaluated them as a random sample of the whole.

Each chapter follows the same structure i.e., an introductory text followed by tables of cross referencing and sometimes other data. For example Chapter 3 "Aldehydes and Ketones", although focussed on tobacco and tobacco smoke contains much information on the isola- tion, derivatization and characterisation of these compounds which is applicable to other fields such as a study of indoor air quality - a subject not unrelated to tobacco smoke.

I read the section on nicotine and other tobacco alkaloids (p.780 et sec.) with interest (and nostalgia), and was pleased to see cotinine classified as 'a common alkaloid found in smoke...' Many still believe that this compound is solely a metabolite of nicotine in vivo. Data in tables is restricted to CAS number and structure but have wider uses than just tobacco.

Chapter 20 on Metallic and Nonmetallic Elements proved somewhat of a disappointment. To date some 80 elements have been detected in tobacco yet this is a short chapter concentrating on elements only, with almost no information on organometallic compounds in tobacco smoke (formed by pyrolytic synthesis?). For example arsenic transfer from tobacco to smoke is reported to be $3.5 \%$ giving around $0.02 \mu \mathrm{g} / \mathrm{cig}$ 'measured as arsenic' - no mention of speciation yet I would anticipate a possibility of methylation. Two other chapters caught my eye. Chapter 21 on Pesticides and Chapter 26 on Carcinogenicity were of interest because of their topicality. In Chapter 21 I was surprised to read of the paucity of information on synthetic pesticide transfer rates from tobacco to smoke and the complete lack of information on the transfer of naturally occurring pesticides and plant growth regulators. Presumably 'if it's natural it must be healthy 'still applies? Chapter 26 on carcinogenic activity was more comprehensive and thus reassuring. I particularly liked the balanced (Assertion vs. Contradiction) approach to this highly emotive topic as it is essential that such important areas of impact on human health are discussed and dealt with objectively. For example, Table XXVI 12 (p1224) should be studied by all barbeque addicts.

I doubt whether this book will find its way into many private libraries but as a reference text on a laboratory shelf it will prove essential and take its place alongside the other CRC publications. Although focussed on the tobacco industry the data will be equally relevant and applicable to other industries such as cosmetics, perfumes and flavours. Indeed anywhere that products have to look good, smell good and taste good.

M. Cooke 